\title{
Vitamin C and thiamine for sepsis: time to go back to fundamental principles
}

\author{
Tomoko Fujii ${ }^{1} 2^{*} \mathbb{D}$, Robert Fowler ${ }^{3}$ and Jean-Louis Vincent ${ }^{4}$
}

๑ 2020 Springer-Verlag GmbH Germany, part of Springer Nature

Vitamin C plays a vital role in human physiology. For decades, intriguing research has highlighted the possibility of acute effects of vitamin $\mathrm{C}$ deficiency in patients with sepsis [1]. Vitamin $C$ is a mediator of endothelial function through its co-factor role with many enzymes (including one that catalyses the conversion of dopamine to noradrenaline). Vitamin $\mathrm{C}$ also works as an antioxidant by scavenging free radicals (Fig. 1). In animal models of sepsis, exogenous vitamin $C$ increases perfused capillary density and arteriolar vasoconstrictor responsiveness, implying its possible role in treating vasoplegic status in sepsis [2].

The synergistic effect of hydrocortisone and vitamin $\mathrm{C}$ has also been suggested as vitamin $\mathrm{C}$ helps to reduce oxidised glucocorticoid receptors, and glucocorticoids induce the expression of a vitamin $C$ transporter [2]. Thiamine, which is a coenzyme for the oxidation of pyruvate to acetyl-CoA in the Krebs cycle, is also depleted in some patients with sepsis [3]. Thus, thiamine has been suggested as another treatment option for sepsis.

The combination of vitamin $\mathrm{C}$, thiamine, and hydrocortisone has recently been advocated as a treatment for patients with septic shock, largely based upon a singlecentre before-after study [4]. This suggestion has provoked substantial global debate on incorporation into clinical practice ahead of assessing the balance of benefits and harms in valid clinical trials that comprise a contemporaneous control group with potentially confounding factors balanced between study arms.

In this issue of Intensive Care Medicine, results from the ATESS trial, a double-blind RCT of vitamin C and

\footnotetext{
*Correspondence: tofujii-tky@umin.net

${ }^{1}$ Intensive Care Unit, Jikei University Hospital, 3-19-18, Nishi-Shimbashi, Minato-ku, Tokyo 105-8461, Japan
}

Full author information is available at the end of the article thiamine for septic shock are reported [5]. The trial was conducted in four emergency departments in South Korea. The trial was designed to assess the effect of intravenous vitamin $C(50 \mathrm{mg} / \mathrm{kg}$, max $3 \mathrm{~g} /$ dose, every $12 \mathrm{~h})$ and thiamine (200 mg, every $12 \mathrm{~h}$ ) for $48 \mathrm{~h}$ compared with placebo on organ dysfunction. A sample size of 116 was calculated to have $80 \%$ power to detect a difference of 1.5 points in the change of SOFA score at $72 \mathrm{~h}$ of enrolment.

Subsequently, 111 of 116 randomised patients were included in the analysis. There was no difference in delta SOFA score [median of differences, $0(-2-1), p=0.96$ ]. None of the secondary outcomes, including mortality, vasopressor dependency, need for mechanical ventilation or renal replacement therapy, and inflammatory biomarkers, indicated a beneficial effect of the intervention.

The allocation sequence and randomisation were concealed appropriately, and the major co-intervention with hydrocortisone was well-balanced between the groups, indicating likely minimal risk of bias due to confounding. The intervention was provided to patients at around $3 \mathrm{~h}$ after meeting the standard criteria of septic shock, and plasma levels of vitamin $C$ and thiamine at $72 \mathrm{~h}$ of enrolment showed clear separation of the two groups.

The trial was well-designed from several perspectives. First, early administration of the intervention at emergency departments, along with early administration of antibiotics and vasopressors, strengthened the potential for this trial to detect early treatment-specific effects while minimising variability in outcomes due to variations in the standard of care for septic shock.

While an intervention of vitamin $C$, thiamine and hydrocortisone has been advocated as a triple-cocktail therapy [4], this trial tested the combination of vitamin $\mathrm{C}$ and thiamine, alongside clinician-determined appropriate treatment with hydrocortisone-balanced between

\section{Springer}




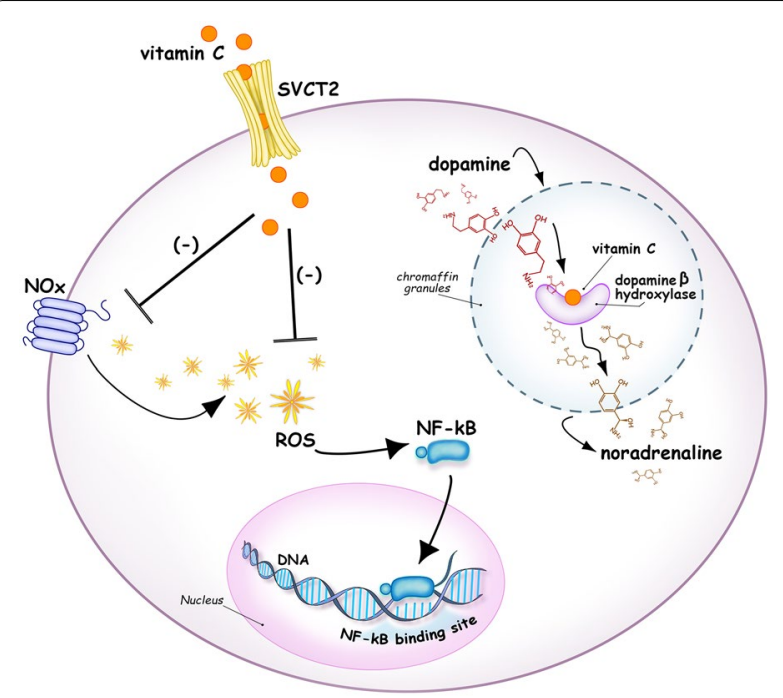

Fig. 1 A diagram of vitamin $C$ roles from in vitro or in vivo studies. Vitamin C is taken up into cells via SVCT2 and scavenge ROS and downregulate NOx. In adrenomedullary chromaffin cells, dopamine is translocated to chromaffin granules and is converted to noradrenaline. The conversion is catalysed by dopamine $\beta$-hydroxylase, where vitamin $\mathrm{C}$ works as a cofactor. SVCT2 sodium-vitamin $C$ cotransporter $2, R O S$ reactive oxygen species, $N O x$ NADPH oxidase. $N F-k B$ nuclear factor-kappa B

study arms, allowing attribution of effects to vitamin $C$ and thiamine.

Several limitations should be noted. First, the duration of the intervention was $48 \mathrm{~h}$, and the primary follow-up period was $72 \mathrm{~h}$, which might be short for the effects to be detected. The pharmacokinetics of intravenous vitamin $C$ in critically ill patients have been tested with $1 \mathrm{~g} /$ dose or $5 \mathrm{~g} /$ dose every $12 \mathrm{~h}$ for $48 \mathrm{~h}$ achieving normal or supranormal trough concentrations; however, a few patients in both groups still had hypovitaminosis at $96 \mathrm{~h}$ [6].

In another RCT, $1.5 \mathrm{~g}$ of intravenous vitamin $\mathrm{C}$ was administered every six hours [7]. The nested cohort study found that the plasma concentration of vitamin $\mathrm{C}$ was kept in the supranormal range at $6 \mathrm{~h}$, and the half-life of the first dose was $4.3 \mathrm{~h}$ [8]. This raises the possibility that vitamin $C$ levels could have been sub-optimal between dosing in the ATESS trial, and could fall beyond the initial treatment period, both leading to a risk of missing a treatment effect.

The measurement of thiamine is more difficult. There is no universal method to measure thiamine as it can exist in various forms, i.e., free-thiamine, thiamine monophosphate, thiamine pyrophosphate, thiamine triphosphate, each requiring somewhat unique methods [9]. In this trial, thiamine deficiency was defined as serum levels $<66.1 \mathrm{nmol} / \mathrm{L}$ [5], a higher threshold than in some previous studies [3, 10]. Finding an optimal therapeutic range and the optimal method of monitoring thiamine concentration would benefit from additional research.

Since the first report of a large effect size with triple therapy [4], more than 20 trials have been planned or are underway. So far, two trials assessing the effect of vitamin $\mathrm{C}$ and thiamine while controlling the use of hydrocortisone as a mandated co-intervention, or balanced by randomisation, have shown no significant effect $[5,7]$. Three trials evaluating vitamin $\mathrm{C}$, thiamine and hydrocortisone, including the recently published ACTS trial, have demonstrated an inconsistent effect upon the vasopressor requirement and SOFA score $[11,12]$. Nevertheless, increasing literature has better defined the role of corticosteroids for septic shock $[13,14]$, highlighting that treatment with vitamin $\mathrm{C}$ and/or thiamine, should likely be independently investigated.

Furthermore, the sample size of the ATESS trial and recent ones are relatively small $[5,11]$, which often produce fragile results. However, fragile results still influence opinions, in this case, on a contemporary controversy. The numerous ongoing trials would likely better serve patients and practitioners if there were attempts to define common interventions and outcomes, to allow meta-analytic combination of trials; and/or if larger collaborative trials could occur from the outset.

Until larger sample sizes improve the precision of an effect size across populations and sub-populations of septic patients, the results of the ATESS trial and others counsel us to remain focused on the fundamental principles in treating sepsis-prompt and measured resuscitation that includes early appropriate empiric antibiotics and source control-while subjecting new therapies to methodologically rigorous clinical trials [15].

\section{Author details \\ ${ }^{1}$ Intensive Care Unit, Jikei University Hospital, 3-19-18, Nishi-Shimbashi, Minato-ku, Tokyo 105-8461, Japan. ${ }^{2}$ Australian and New Zealand Intensive Care Research Centre, Monash University School of Public Health and Pre- ventive Medicine, Melbourne, VIC, Australia. ${ }^{3}$ Department of Medicine and Department of Critical Care Medicine, Sunnybrook Health Sciences Cen- tre, Toronto, ON, Canada. ${ }^{4}$ Department of Intensive Care, Erasme University Hospital, Brussels, Belgium.}

\section{Author contributions}

All authors contributed substantially to the conception of the editorial and drafted or provided critical revision of the article and provided final approval of the version submitted for publication.

\section{Compliance with ethical standards}

Conflicts of interest

TF was an investigator of VITAMINS trial. All authors declare no COI. 


\section{Publisher's Note}

Springer Nature remains neutral with regard to jurisdictional claims in published maps and institutional affiliations.

Received: 10 August 2020 Accepted: 4 September 2020

Published online: 18 September 2020

\section{References}

1. Moskowitz A, Andersen LW, Huang DT et al (2018) Ascorbic acid, corticosteroids, and thiamine in sepsis: a review of the biologic rationale and the present state of clinical evaluation. Crit Care 22:283

2. Fujji T, Deane AM, Nair P (2020) Metabolic support in sepsis: corticosteroids and vitamins: the why, the when, the how. Curr Opin Crit Care 26(4):363-368

3. Donnino MW, Carney E, Cocchi MN, Barbash I, Chase M, Joyce N et al (2010) Thiamine deficiency in critically ill patients with sepsis. J Crit Care 25(4):576-581

4. Marik PE, Khangoora V, Rivera R, Hooper MH, Catravas J (2017) Hydrocortisone, Vitamin $\mathrm{C}$ and thiamine for the treatment of severe sepsis and septic shock: a retrospective before-after study. Chest 151(6):1229-1238

5. Hwang SY, Ryoo SM, Park JE, Jo YH, Jang D-H, Suh GJ et al (2020) Combination therapy of vitamin $\mathrm{C}$ and thiamine for septic shock: a multicentre, double-blind, randomized Controlled Study. Intensive Care Med 2:167-345

6. de Grooth H-J, Manubulu-Choo W-P, Zandvliet AS et al (2018) Vitamin C pharmacokinetics in critically III patients. Chest 153(6):1368-1377
7. Fujii T, Luethi N, Young PJ, Frei DR, Eastwood GM, French CJ et al (2020) Effect of Vitamin C, hydrocortisone, and thiamine vs hydrocortisone alone on time alive and free of vasopressor support among patients with septic shock: the vitamins randomized clinical trial. JAMA 323(5):423-431

8. Hudson EP, Collie JT, Fujii T, Luethi N, Udy AA, Doherty S et al (2019) Pharmacokinetic data support 6-hourly dosing of intravenous vitamin C to critically ill patients with septic shock. Crit Care Resusc 21(4):236-242

9. Collie JTB, Greaves RF, Jones OAH, Lam Q, Eastwood GM, Bellomo R (2017) Vitamin B1 in critically ill patients: Needs and challenges. Clin Chem Lab Med 55(11):1652-1668

10. Costa NA, Gut AL, de Souza DM, Pimentel JA, Cozzolino SM, Azevedo PS et al (2014) Serum thiamine concentration and oxidative stress as predictors of mortality in patients with septic shock. J Crit Care 29(2):249-252

11. Fujii T, Udy AA (2020) Additional trials of vitamin C in septic shock: a bag of mixed fruit. Chest 158(1):13-14

12. Moskowitz A, Huang DT, Hou PC et al (2020) ACTS clinical trial investigators effect of ascorbic acid, corticosteroids, and thiamine on organ injury in septic shock: the acts randomized clinical trial. JAMA 324(7):642-650

13. Annane D, Renault A, Brun-Buisson C et al (2018) Hydrocortisone plus fludrocortisone for adults with septic shock. N Engl J Med 378(9):809-818

14. Venkatesh B, Finfer S, Cohen J et al (2018) Adjunctive glucocorticoid therapy in patients with septic shock. N Engl J Med 378(9):797-808

15. Rhodes A, Evans L, Alhazzani W et al (2016) Surviving sepsis campaign: International guidelines for management of sepsis and septic shock: 2016. Intensive Care Med 43:304-377 